\title{
Combined procedure of the transcervical radiofrequency ablation (TRFA) system and surgical hysteroscopy. Increased risk or safe procedure?
}

\author{
Elvin Piriyev ${ }^{1}$, Sven Schiermeier ${ }^{2}$, Thomas Römer ${ }^{3}$ \\ ${ }^{1}$ Department of Obstetrics and Gynecology, Academic Hospital Cologne Weyertal University of Cologne, University Witten-Herdecke, Germany \\ ${ }^{2}$ Department of Obstetrics and Gynecology, University Witten-Herdecke, Marien-Hospital, Witten, Germany \\ ${ }^{3}$ Department of Obstetrics and Gynecology, Academic Hospital, Cologne Weyertal University of Cologne, Germany
}

\begin{abstract}
Introduction: The advantage of transcervical radiofrequency ablation (TRFA) is that it is minimally invasive, incision-free, and treats a wide spectrum of fibroids, including those that are not accessible by surgical hysteroscopy (FIGO 3, 4, 5, 6, and 2-5). However, there are no publications describing a combined procedure of operative hysteroscopy and TRFA yet, so it was still unknown whether a combined procedure is associated with additional risks.

Aim: To report the combined technique of transcervical intrauterine radiofrequency ablation of fibroids and surgical hysteroscopy.

Material and methods: Our study was designed to show the results of our case series with 21 patients. The retrospective study included only patients who were treated with the combined procedure of surgical hysteroscopy with fibroid and/or endometrial resection and fibroid ablation using the Sonata System.

Results: The combined procedure was performed without any complications in all cases. Two days after surgery, no increased morbidity was observed compared to only conventional surgical hysteroscopy and/or therapy with the TRFA. All patients were satisfied with the procedure. No late complications were observed within the first 6 months postoperatively. Seventeen patients with bleeding symptoms were asked about their subjective assessment of improvement. Fifteen patients reported significant improvement in symptoms and 1 patient reported only minimal improvement. Only 1 patient, who underwent TRFA and endometrial resection, did not report any improvement. No increase in symptoms was observed. Conclusions: Although TRFA is an approved method, it is not yet widely used worldwide. The combined procedure has been rarely used. The aim of our work is to show through our case series that transcervical radiofrequency ablation can be combined with surgical hysteroscopy for fibroid and/or endometrial resection without any additional risk.
\end{abstract}

Key words: ablation, fibroid, Sonata, surgical hysteroscopy, transcervical radiofrequency ablation, uterine bleeding.

\section{Introduction}

Uterine fibroids are the most common benign tumors in women, with an estimated prevalence ranging from $4.5 \%$ to $68.6 \%$ [1]. Fibroids can cause various symptoms, with hypermenorrhea being the main symptom at 40-54\%, followed by dysmenor- rhea (approximately 28\%) and lower abdominal pain (approximately 15\%) [2, 3].

Among all fibroid types, submucosal fibroids are associated with the most severe symptoms, including heavy bleeding disorder, dysmenorrhea, infertility, and repeated abortions [4]. In addition, submucosal

\section{Address for correspondence}

Dr. med. Elvin Piriyev, University Witten-Herdecke, Department of Obstetrics and Gynecology, Academic Hospital Cologne Weyertal

University of Cologne, Germany, e-mail: piriyev.elvins@gmail.com 
fibroids are the most common cause of iron deficiency anemia in premenopausal women [4]. However, intramural fibroids, especially those located close to the cavum, can equally cause bleeding problems [5].

Intramural fibroids close to the cavum and submucosal fibroids often present with symptoms such as vaginal bleeding disorders (e.g., hypermenorrhea, dysmenorrhea) requiring a targeted therapy [5].

Various invasive and radical therapies, such as a hysterectomy, are used in the treatment of fibroids. The most common treatment for symptomatic fibroids is still hysterectomy, and fibroids are the main indication for hysterectomy [6-9]. However, in a survey of 968 women with symptomatic fibroids, $79 \%$ of women wanted to avoid invasive therapy and $51 \%$ desired organ-preserving therapy [9].

Bipolar surgical hysteroscopy is usually the method of choice for submucosal fibroids [5]. However, this method is either not possible for intramural fibroids or has a higher risk of complications [10]. Therefore, transcervical radiofrequency ablation (TRFA) was developed. The advantage of this method is that it is minimally invasive, incision-free, and treats a wide spectrum of fibroids, including those that are not accessible by surgical hysteroscopy (FIGO 3, 4, 5, 6, and 2-5) [10].

Transcervical radiofrequency ablation has FDA (U.S. Food and Drug Administration) approval for diagnostic intrauterine imaging and transcervical treatment of symptomatic uterine fibroids, including those that are associated with heavy menstrual bleeding. The system also has a CE mark ("Conformité Européenne", French for "European Conformity") in the European Union [11-13].

In recent years, transcervical endometrial resection has been established as an alternative for a hysterectomy in the treatment of bleeding disorders. The advantages are fewer complications and rapid recovery compared to a hysterectomy. However, in women with uterine fibroids, there is a reintervention rate of $18 \%$ that results in a hysterectomy [14].

There are no publications describing a combined procedure of operative hysteroscopy and TRFA yet, so it was still unknown whether a combined procedure is associated with additional risks. Therefore, our study was designed to show the results of our case series.

\section{Aim}

There are no publications describing a combined procedure of operative hysteroscopy and TRFA yet, so it was still unknown whether a combined procedure is associated with additional risks. The aim was to report the combined technique of transcervical intrauterine radiofrequency ablation of fibroids and surgical hysteroscopy.

\section{Material and methods}

Transcervical radiofrequency ablation (primarily known as VizAblate, then Sonata Gynesonics System) was introduced in our department in August 2011. Since then, 151 patients have been treated using this method up to the time of this analysis, with the number of patients steadily increasing each year. The retrospective study included only patients (14\%) who were treated with the combined procedure of surgical hysteroscopy with fibroid and/or endometrial resection and fibroid ablation using the Sonata System in the Academic Hospital Cologne Weyertal.

The diagnosis of fibroids was performed by transvaginal sonography.

A total of 21 patients were included in the study and 58 fibroids were treated.

The combined therapy was performed without any complications in all cases. During the surgery, the Sonata System could be positioned without any problems. After fixation of the fibroid with the central spike ("introducer"), the ablation zone and safety zone were adjusted and the electrodes were inserted. Subsequently, after rechecking the safety zone, the ablation could be performed once a temperature of $105^{\circ} \mathrm{C}$ was reached. Bipolar operative hysteroscopy was performed in the usual manner. All patients were discharged from the hospital feeling well.

Since the smallest ablation zone with the Sonata System has a diameter of $2 \mathrm{~cm}$, the following order was applied to fibroids being treated simultaneously with both methods:

- for FIGO 1 and 2 fibroids $<3 \mathrm{~cm}$, first ablation with the Sonata System followed by resection with bipolar operative hysteroscopy.

- for fibroids $>3 \mathrm{~cm}$, first bipolar operative hysteroscopy with fibroid resection followed by ablation with the Sonata System.

In other cases, the order can be determined by the surgeon.

Follow-up was done either by standard progress monitoring at 6 months after surgery or by telephone contact. Intraoperative and postoperative complications, postoperative morbidity, and improvement in bleeding symptoms were recorded. 


\section{Ethical approval}

According to $15 \S$ of the professional code of the North Rhine Medical Association, neither advice nor an ethics vote is necessary for a retrospective study.

\section{Results}

The age of the patients ranged from 28 to 51 years, with a mean age of 43 years. The indication for surgery was hypermenorrhea in 16 cases and prolonged bleeding in 3 cases. Two patients presented with fertility desire and already had two failed surgical hysteroscopies.

A total of 58 fibroids were detected and treated. Fibroids were divided according to the FIGO classification and depending on their size (Table I). The majority of fibroids (46.5\%) consisted of FIGO 0 and 1 fibroids. $48.2 \%$ of fibroids were up to $2 \mathrm{~cm}$ in diameter (Table I).

The combined procedure was divided into three groups: the first group consisted of the Sonata System and fibroid resection, the second group of the Sonata System and endometrial resection, and the

Table I. Overview of fibroids

\begin{tabular}{|lccc|}
\hline FIGO & $N$ & Size $[\mathrm{cm}]$ & $N$ \\
\hline FIGO 0 and 1 & 27 & $0-2$ & 28 \\
\cline { 1 - 2 } FIGO 2 & 6 & $2.1-4$ & 23 \\
\hline FIGO 3 & 7 & $4.1-6$ & 5 \\
\cline { 1 - 2 } FIGO 4 & 2 & & \\
\cline { 1 - 2 } FIGO 5 & 5 & $6.1-8$ & 2 \\
\cline { 1 - 2 } FIGO 2-5 & 11 & & \\
\hline
\end{tabular}

$N-$ number of fibroids.

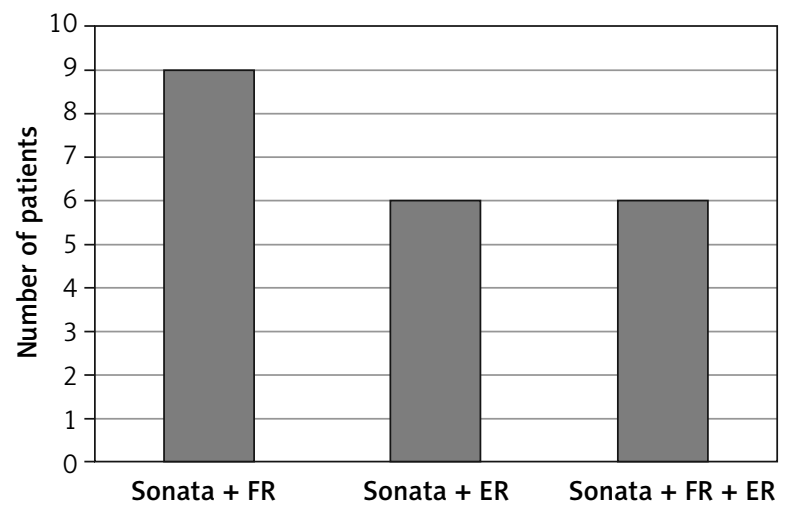

Figure 1. Distribution of patients depending on the method performed

$F R$ - fibroid resection, $E R$ - endometrium resection. third group of the Sonata System and fibroid and endometrial resection (Figure 1).

Thirty-two fibroids were treated with the Sonata System and 26 fibroids with surgical hysteroscopy, of which 8 fibroids were both ablated with the Sonata System and partially resected using surgical hysteroscopy.

Fourteen patients with completed family planning had simultaneous endometrial resection, of which 9 patients had both endometrial and fibroid resection and 5 patients had only an endometrial resection in addition to the Sonata System.

The shortest ablation time with the Sonata System was $1 \mathrm{~min}$ and $24 \mathrm{~s}$ and the longest time was $13 \mathrm{~min}$ and $42 \mathrm{~s}$ (Table II).

The combined procedure was performed without any complications in all cases. Two days after surgery, no increased morbidity was observed compared to only conventional surgical hysteroscopy and/or therapy with the Sonata System. All patients were satisfied with the procedure.

A follow-up was carried out with 19 patients. No late complications were observed within the first 6 months postoperatively. Seventeen patients with bleeding symptoms were asked about their subjective assessment of improvement. Fifteen patients reported significant improvement in symptoms and 1 patient reported only minimal improvement. Only one patient, who underwent treatment with the Sonata System and endometrial resection, did not report any improvement. No increase in symptoms was observed.

Follow-up was not long enough with two patients and with 1 patient there was no follow-up.

\section{Discussion}

Nowadays, there are several options in the treatment of fibroids, from drug therapy to a radical approach such as a hysterectomy [6-9, 11, 15-17]. Fibroid therapy depends on organ findings (FIGO type, size, number of fibroids), severity of symptoms, patient's life stage (fertile, peri- or postmenopausal life

Table II. Time of ablation with Sonata System

\begin{tabular}{|lc|}
\hline Time of ablation [min] & Number of myomas \\
\hline $0-2$ & 10 \\
\hline $2.1-4$ & 17 \\
\hline $6.1-8$ & 4 \\
\hline $12-14$ & 1 \\
\hline
\end{tabular}


stage), health risk factors, medical contraindications and patient's wishes.

A survey was conducted in which 968 women with symptomatic fibroids participated. Seventy-nine percent of women wanted to avoid invasive therapy and $51 \%$ desired organ-preserving therapy [9].

The most common treatment for symptomatic fibroids remains a hysterectomy, and fibroids represent the main indication for a hysterectomy [6-9].

For submucosal fibroids, the method of choice is usually a bipolar surgical hysteroscopy [5]. However, the hysteroscopic approach can be particularly difficult for large $(>3 \mathrm{~cm})$, for multiple $(n>3)$, and for type 2 fibroids, and there is a $20-50 \%$ chance that at least one additional resection will still be required for a complete fibroid resection $[5,18,19]$. The intraoperative complication risk of a hysteroscopy is low [20].

Surgical hysteroscopy with endometrial resection in women with bleeding symptoms is widely used [14]. Christoffersen et al. found that endometrial resection in a uterus with FIGO 2 fibroid and a size of $>3.5 \mathrm{~cm}$ significantly increased the risk of reintervention, usually a hysterectomy, compared to a uterus with FIGO 0 fibroid and a size of $<3.5 \mathrm{~cm}$ [14].

While a hysterectomy and other more invasive options remain widely used, there remains a need for less invasive organ-preserving fibroid treatment that is incision-free and not limited to a narrow range of myoma types (FIGO types 0 and 1 and small type 2 myomas), as is the case with surgical hysteroscopy [11-13]. Therefore, transcervical radiofrequency ablation (TRF) was developed. TRF has been proven to be a safe and effective method [11, 15-17].

In Toub's study, 94\% (99/105) of patients were satisfied with TRF and 88\% (92/105) reported an improvement in bleeding symptoms [11]. The success rate in our case series was 94\% (16/17).

In the VITALITY study, the long-term results after TRF were published. The reintervention rate was $11.8 \%$ [21]. In a meta-analysis with more than 35000 patients, the reintervention rates of different methods were shown over 3 years. The reintervention rate was $9 \%$ after open myomectomy, $11 \%$ after a laparoscopic myomectomy, $17 \%$ after UAE (uterine artery embolization), $21 \%$ after a hysteroscopic fibroid resection, and $24 \%$ after an endometrial ablation [22].

\section{Conclusions}

The combined procedure of the TRFA and surgical hysteroscopy is a simple, minimally invasive, rapid and successful method in the treatment of bleeding disorders in women with uterine fibroids.

Although TRFA is an approved method, it is not yet widely used worldwide. The combined procedure has been rarely used. There is no publication describing the combined procedure yet. For this reason, our analysis was carried out. The aim of our work is to show through our case series that the transcervical radiofrequency ablation can be combined with surgical hysteroscopy for fibroid and/or endometrial resection without any additional risk. A prospective study will be initiated.

\section{Conflict of interest}

EP declares no conflict of interest. SS and TR are consultants for the Sonata System. The authors report no other conflicts of interest in this work.

\section{References}

1. Stewart EA, Cookson CL, Gandolfo RA, Schulze-Rath R. Epidemiology of uterine fibroids: a systematic review. BJOG 2017; 124: 1501-12.

2. Foth D, Röhl FW, Friedrich C, et al. Symptoms of uterine myomas: data of an epidemiological study in Germany. Arch Gynecol Obstet 2017; 295: 415-26.

3. Zimmermann A, Bernuit D, Gerlinger C, et al. Prevalence, symptoms and management of uterine fibroids: an international internet-based survey of 21,746 women. BMC Womens Health 2012; 12: 6.

4. Yang JH, Chen MJ, Chen CD, et al. Impact of submucous myoma on the severity of anemia. Fertil Steril 2011; 95: 1769-72.

5. Römer T. Medical treatment of fibroids. De Gruyter 2019.

6. Farquhar CM, Steiner CA. Hysterectomy rates in the United States 1990-1997. Obstet Gynecol 2002; 99: 229-34.

7. Wise LA, Palmer JR, Stewart EA, Rosenberg L. Age-specific incidence rates for self-reported uterine leiomyomata in the Black Women's Health Study. Obstet Gynecol 2005; 105: 563-8.

8. Downes E, Sikirica V, Gilabert-Estelles J, et al. The burden of uterine fibroids in five European countries. Eur J Obstet Gynecol Reprod Biol 2010; 152: 96-102.

9. Borah BJ, Nicholson WK, Bradley L, Stewart EA. The impact of uterine leiomyomas: a national survey of affected women. Am J Obstet Gynecol 2013; 209: 319e1-20.

10. Downes E, Sikirica V, Gilabert-Estelles J, et al. The burden of uterine fibroids in five European countries. Eur J Obstet Gynecol Reprod Biol 2010; 152: 96-102.

11. Toub DB. A new paradigm for uterine fibroid treatment: transcervical, intrauterine sonography-guided radiofrequency ablation of uterine fibroids with the Sonata System. Curr Obstet Gynecol Rep 2017; 6: 67-73.

12. Bongers M, Brölmann H, Gupta J, et al. Transcervical, intrauterine ultrasound-guided radiofrequency ablation of uterine fibroids with the VizAblate ${ }^{\circledR}$ System: three- and six-month 
endpoint results from the FAST-EU study. Gynecol Surg 2015; 12: 61-70.

13. Chudnoff S, Guido R, Roy K, et al. Ultrasound-guided transcervical ablation of uterine leiomyomas. Obstet Gynecol 2019; 133 13-22.

14. Christoffersen C, Kahr HS, Sørensen SS. Impact of myomas on the results of transcervical resection of the endometrium. J Minim Invasive Gynecol 2014; 21: 811-7.

15. Bradley LD, Pasic RP, Miller LE. Clinical performance of radiofrequency ablation for treatment of uterine fibroids: systematic review and meta-analysis of prospective studies. J Laparoendosc Adv Surg Tech A 2019; 29: 1507-17.

16. Iversen H, Dueholm M. Radiofrequency thermal ablation for uterine myomas: long-term clinical outcomes and reinterventions. J Minim Invasive Gynecol 2017; 24: 1020-8.

17. Lin L, Ma H, Wang J, et al. Quality of life, adverse events, and reintervention outcomes after laparoscopic radiofrequency ablation for symptomatic uterine fibroids: a meta-analysis. J Minim Invasive Gynecol 2019; 26: 409-16.

18. Vercellini P, Cortesi I, Oldani S, et al. The role of transvaginal ultrasonography and outpatient diagnostic hysteroscopy in the evaluation of patients with menorrhagia. Hum Reprod 1997; 12: 1768-71.

19. Wamsteker K, Emanuel MH, de Kruif JH. Transcervical hysteroscopic resection of submucous fibroids for abnormal uterine bleeding: results regarding the degree of intramural extension. Obstet Gynecol 1993; 82: 736-40.

20. Römer T. Hysteroskopischer Wegweiser für Gynäkologen. $2 \mathrm{Au}$ flage. De Gruyter 2008.

21. Garza-Leal JG. Long-term clinical outcomes of transcervical radiofrequency ablation of uterine fibroids: the VITALITY study. J Gynecol Surg 2019; 35: 19-23.

22. Davis MR, Soliman AM, Castelli-Haley J, et al. Reintervention rates after myomectomy, endometrial ablation, and uterine artery embolization for patients with uterine fibroids. J Womens Health 2018; 27: 1204-14.

Received: 15.12.2021, accepted: 11.01.2022. 